\title{
A note on three types of quasisymmetric functions
}

\author{
T. Kyle Petersen \\ Department of Mathematics \\ Brandeis University, Waltham, MA, USA \\ tkpeters@brandeis.edu
}

Submitted: Aug 8, 2005; Accepted: Nov 14, 2005; Published: Nov 22, 2005

Mathematics Subject Classifications: 05E99, 16S34

\begin{abstract}
In the context of generating functions for $P$-partitions, we revisit three flavors of quasisymmetric functions: Gessel's quasisymmetric functions, Chow's type B quasisymmetric functions, and Poirier's signed quasisymmetric functions. In each case we use the inner coproduct to give a combinatorial description (counting pairs of permutations) to the multiplication in: Solomon's type A descent algebra, Solomon's type B descent algebra, and the Mantaci-Reutenauer algebra, respectively. The presentation is brief and elementary, our main results coming as consequences of $P$-partition theorems already in the literature.
\end{abstract}

\section{Quasisymmetric functions and Solomon's descent algebra}

The ring of quasisymmetric functions is well-known (see [12], ch. 7.19). Recall that a quasisymmetric function is a formal series

$$
Q\left(x_{1}, x_{2}, \ldots\right) \in \mathbb{Z}\left[\left[x_{1}, x_{2}, \ldots\right]\right]
$$

of bounded degree such that the coefficient of $x_{i_{1}}^{\alpha_{1}} x_{i_{2}}^{\alpha_{2}} \cdots x_{i_{k}}^{\alpha_{k}}$ is the same for all $i_{1}<$ $i_{2}<\cdots<i_{k}$ and all compositions $\alpha=\left(\alpha_{1}, \alpha_{2}, \ldots, \alpha_{k}\right)$. Recall that a composition of $n$, written $\alpha \models n$, is an ordered tuple of positive integers $\alpha=\left(\alpha_{1}, \alpha_{2}, \ldots, \alpha_{k}\right)$ such that $|\alpha|=\alpha_{1}+\alpha_{2}+\cdots+\alpha_{k}=n$. In this case we say that $\alpha$ has $k$ parts, or $\# \alpha=k$. We can put a partial order on the set of all compositions of $n$ by reverse refinement. The covering relations are of the form

$$
\left(\alpha_{1}, \ldots, \alpha_{i}+\alpha_{i+1}, \ldots, \alpha_{k}\right) \prec\left(\alpha_{1}, \ldots, \alpha_{i}, \alpha_{i+1}, \ldots, \alpha_{k}\right) .
$$

Let $\mathcal{Q}$ sym $_{n}$ denote the set of all quasisymmetric functions homogeneous of degree $n$. The ring of quasisymmetric functions can be defined as $\mathcal{Q} s y m:=\bigoplus_{n \geq 0} \mathcal{Q}$ sym $_{n}$, but our focus will stay on the quasisymmetric functions of degree $n$, rather than the ring as a whole. 
The most obvious basis for $\mathcal{Q} \mathrm{sm}_{n}$ is the set of monomial quasisymmetric functions, defined for any composition $\alpha=\left(\alpha_{1}, \alpha_{2}, \ldots, \alpha_{k}\right) \models n$,

$$
M_{\alpha}:=\sum_{i_{1}<i_{2}<\cdots<i_{k}} x_{i_{1}}^{\alpha_{1}} x_{i_{2}}^{\alpha_{2}} \cdots x_{i_{k}}^{\alpha_{k}}
$$

We can form another natural basis with the fundamental quasisymmetric functions, also indexed by compositions,

$$
F_{\alpha}:=\sum_{\alpha \preccurlyeq \beta} M_{\beta},
$$

since, by inclusion-exclusion we can express the $M_{\alpha}$ in terms of the $F_{\alpha}$ :

$$
M_{\alpha}=\sum_{\alpha \preccurlyeq \beta}(-1)^{\# \beta-\# \alpha} F_{\beta} .
$$

As an example,

$$
F_{(2,1)}=M_{(2,1)}+M_{(1,1,1)}=\sum_{i<j} x_{i}^{2} x_{j}+\sum_{i<j<k} x_{i} x_{j} x_{k}=\sum_{i \leq j<k} x_{i} x_{j} x_{k} .
$$

Compositions can be used to encode descent classes of permutations in the following way. Recall that a descent of a permutation $\pi \in \mathfrak{S}_{n}$ is a position $i$ such that $\pi_{i}>\pi_{i+1}$, and that an increasing run of a permutation $\pi$ is a maximal subword of consecutive letters $\pi_{i+1} \pi_{i+2} \cdots \pi_{i+r}$ such that $\pi_{i+1}<\pi_{i+2}<\cdots<\pi_{i+r}$. By maximality, we have that if $\pi_{i+1} \pi_{i+2} \cdots \pi_{i+r}$ is an increasing run, then $i$ is a descent of $\pi$ (if $i \neq 0$ ), and $i+r$ is a descent of $\pi$ (if $i+r \neq n$ ). For any permutation $\pi \in \mathfrak{S}_{n}$ define the descent composition, $C(\pi)$, to be the ordered tuple listing the lengths of the increasing runs of $\pi$. If $C(\pi)=\left(\alpha_{1}, \alpha_{2}, \ldots, \alpha_{k}\right)$, we can recover the descent set of $\pi$ :

$$
\operatorname{Des}(\pi)=\left\{\alpha_{1}, \alpha_{1}+\alpha_{2}, \ldots, \alpha_{1}+\alpha_{2}+\cdots+\alpha_{k-1}\right\} .
$$

Since $C(\pi)$ and $\operatorname{Des}(\pi)$ have the same information, we will use them interchangeably. For example the permutation $\pi=(3,4,5,2,6,1)$ has $C(\pi)=(3,2,1)$ and $\operatorname{Des}(\pi)=\{3,5\}$.

Recall ([11], ch. 4.5$)$ that a $P$-partition is an order-preserving map from a poset $P$ to some (countable) totally ordered set. To be precise, let $P$ be any labeled partially ordered set (with partial order $<_{P}$ ) and let $S$ be any totally ordered countable set. Then $f: P \rightarrow S$ is a $P$-partition if it satisfies the following conditions:

1. $f(i) \leq f(j)$ if $i<_{P} j$

2. $f(i)<f(j)$ if $i<_{P} j$ and $i>j$ (as labels)

We let $\mathcal{A}(P)$ (or $\mathcal{A}(P ; S)$ if we want to emphasize the image set) denote the set of all $P$-partitions, and encode this set in the generating function

$$
\Gamma(P):=\sum_{f \in \mathcal{A}(P)} x_{f(1)} x_{f(2)} \cdots x_{f(n)}
$$


where $n$ is the number of elements in $P$ (we will only consider finite posets). If we take $S$ to be the set of positive integers, then it should be clear that $\Gamma(P)$ is always going to be a quasisymmetric function of degree $n$. As an easy example, let $P$ be the poset defined by $3>_{P} 2<_{P} 1$. In this case we have

$$
\Gamma(P)=\sum_{f(3) \geq f(2)<f(1)} x_{f(1)} x_{f(2)} x_{f(3)}
$$

We can consider permutations to be labeled posets with total order $\pi_{1}<_{\pi} \pi_{2}<_{\pi}$ $\cdots<_{\pi} \pi_{n}$. With this convention, we have

$$
\begin{aligned}
\mathcal{A}(\pi)=\{f:[n] \rightarrow S \mid & f\left(\pi_{1}\right) \leq f\left(\pi_{2}\right) \leq \cdots \leq f\left(\pi_{n}\right) \\
& \text { and } \left.k \in \operatorname{Des}(\pi) \Rightarrow f\left(\pi_{k}\right)<f\left(\pi_{k+1}\right)\right\},
\end{aligned}
$$

and

$$
\Gamma(\pi)=\sum_{\substack{i_{1} \leq i_{2} \leq \cdots \leq i_{n} \\ k \in \operatorname{Des}(\pi) \Rightarrow i_{k}<i_{k+1}}} x_{i_{1}} x_{i_{2}} \cdots x_{i_{n}}
$$

It is not hard to verify that in fact we have

$$
\Gamma(\pi)=F_{C(\pi)},
$$

so that generating functions for the $P$-partitions of permutations of $\pi \in \mathfrak{S}_{n}$ form a basis for $\mathcal{Q}_{\text {sym }}$.

We have the following theorem related to $P$-partitions of permutations, due to Gessel $[5]$.

Theorem 1 As sets, we have the bijection

$$
\mathcal{A}(\pi ; S T) \leftrightarrow \coprod_{\sigma \tau=\pi} \mathcal{A}(\tau ; S) \oplus \mathcal{A}(\sigma ; T)
$$

where $S T$ is the cartesian product of the sets $S$ and $T$ with the lexicographic ordering.

Let $X=\left\{x_{1}, x_{2}, \ldots\right\}$ and $Y=\left\{y_{1}, y_{2}, \ldots\right\}$ be two two sets of commuting indeterminates. Then we define the bipartite generating function,

$$
\Gamma(\pi)(X Y)=\sum_{\substack{\left(i_{1}, j_{1}\right) \leq\left(i_{2}, j_{2}\right) \leq \cdots \leq\left(i_{n}, j_{n}\right) \\ k \in \operatorname{Des}(\pi) \Rightarrow\left(i_{k}, j_{k}\right)<\left(i_{k+1}, j_{k+1}\right)}} x_{i_{1}} \cdots x_{i_{n}} y_{j_{1}} \cdots y_{j_{n}} .
$$

We will apply Theorem 1 with $S=T=\mathbb{P}$, the positive integers.

Corollary 1 We have

$$
F_{C(\pi)}(X Y)=\sum_{\sigma \tau=\pi} F_{C(\tau)}(X) F_{C(\sigma)}(Y)
$$


Following [5], we can define a coalgebra structure on $\mathcal{Q} s y m_{n}$ in the following way. If $\pi$ is any permutation with $C(\pi)=\gamma$, let $a_{\alpha, \beta}^{\gamma}$ denote the number of pairs of permutations $(\sigma, \tau) \in \mathfrak{S}_{n} \times \mathfrak{S}_{n}$ with $C(\sigma)=\alpha, C(\tau)=\beta$, and $\sigma \tau=\pi$. Then Corollary 1 defines a

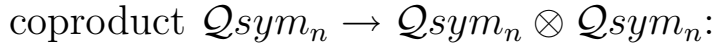

$$
F_{\gamma} \mapsto \sum_{\alpha, \beta \models n} a_{\alpha, \beta}^{\gamma} F_{\beta} \otimes F_{\alpha}
$$

If $\mathcal{Q} s y m_{n}^{*}$, with basis $\left\{F_{\alpha}^{*}\right\}$, is the algebra dual to $\mathcal{Q} s y m_{n}$, then by definition it is equipped with multiplication

$$
F_{\beta}^{*} * F_{\alpha}^{*}=\sum_{\gamma} a_{\alpha, \beta}^{\gamma} F_{\gamma}^{*} .
$$

Let $\mathbb{Z} \mathfrak{S}_{n}$ denote the group algebra of the symmetric group. We can define its dual coalgebra $\mathbb{Z} \mathfrak{S}_{n}^{*}$ with comultiplication

$$
\pi \mapsto \sum_{\sigma \tau=\pi} \tau \otimes \sigma
$$

Then by Corollary 1 we have a surjective homomorphism of coalgebras $\varphi^{*}: \mathbb{Z}_{n}^{*} \rightarrow$ Q $_{\text {sym }}$ given by

$$
\varphi^{*}(\pi)=F_{C(\pi)} .
$$

The dualization of this map is then an injective homomorphism of algebras $\varphi: \mathcal{Q}_{s y m}^{*} \rightarrow$ $\mathbb{Z} \mathfrak{S}_{n}$ with

$$
\varphi\left(F_{\alpha}^{*}\right)=\sum_{C(\pi)=\alpha} \pi
$$

The is image of $\varphi$ is then a subalgebra of the group algebra, with basis

$$
u_{\alpha}:=\sum_{C(\pi)=\alpha} \pi
$$

This subalgebra is well-known as Solomon's descent algebra [10], denoted $\operatorname{Sol}\left(A_{n-1}\right)$. Corollary 1 has then given a combinatorial description to multiplication in $\operatorname{Sol}\left(A_{n-1}\right)$ :

$$
u_{\beta} u_{\alpha}=\sum_{\gamma \models n} a_{\alpha, \beta}^{\gamma} u_{\gamma} .
$$

The above arguments are due to Gessel [5]. We give them here in full detail for comparison with later sections, when we will outline a similar relationship between Chow's type B quasisymmetric functions [4] and $\operatorname{Sol}\left(B_{n}\right)$, and between Poirier's signed quasisymmetric functions [9] and the Mantaci-Reutenauer algebra. 


\section{Type B quasisymmetric functions and Solomon's descent algebra}

The type B quasisymmetric functions can be viewed as the natural objects related to type B $P$-partitions (see [4]). Define the type B posets (with $2 n+1$ elements) to be posets labeled distinctly by $\{-n, \ldots,-1,0,1, \ldots, n\}$ with the property that if $i<_{P} j$, then $-j<_{P}-i$. For example, $-2>_{P} 1<_{P} 0<_{P}-1>_{P} 2$ is a type B poset.

Let $P$ be any type B poset, and let $S=\left\{s_{0}, s_{1}, \ldots\right\}$ be any countable totally ordered set with a minimal element $s_{0}$. Then a type B $P$-partition is any map $f: P \rightarrow \pm S$ such that

1. $f(i) \leq f(j)$ if $i<_{P} j$

2. $f(i)<f(j)$ if $i<_{P} j$ and $i>j$ (as labels)

3. $f(-i)=-f(i)$

where $\pm S$ is the totally ordered set

$$
\cdots<-s_{2}<-s_{1}<s_{0}<s_{1}<s_{2}<\cdots
$$

If $S$ is the nonnegative integers, then $\pm S$ is the set of all integers.

The third property of type B $P$-partitions means that $f(0)=0$ and the set $\{f(i) \mid$ $i=1,2, \ldots, n\}$ determines the map $f$. We let $\mathcal{A}_{B}(P)=\mathcal{A}_{B}(P ; \pm S)$ denote the set of all type B $P$-partitions, and define the generating function for type B $P$-partitions as

$$
\Gamma_{B}(P):=\sum_{f \in \mathcal{A}_{B}(P)} x_{|f(1)|} x_{|f(2)|} \cdots x_{|f(n)|} .
$$

Signed permutations $\pi \in \mathfrak{B}_{n}$ are type B posets with total order

$$
-\pi_{n}<\cdots<-\pi_{1}<0<\pi_{1}<\cdots<\pi_{n} .
$$

We then have

$$
\begin{aligned}
\mathcal{A}_{B}(\pi)=\{f: \pm[n] \rightarrow \pm S \mid & \leq f\left(\pi_{1}\right) \leq f\left(\pi_{2}\right) \leq \cdots \leq f\left(\pi_{n}\right) \\
& f(-i)=-f(i), \\
& \text { and } \left.k \in \operatorname{Des}_{B}(\pi) \Rightarrow f\left(\pi_{k}\right)<f\left(\pi_{k+1}\right)\right\}
\end{aligned}
$$

and

$$
\Gamma_{B}(\pi)=\sum_{\substack{0 \leq i_{1} \leq i_{2} \leq \ldots \leq i_{n} \\ k \in \operatorname{Des}(\pi) \Rightarrow i_{k}<i_{k+1}}} x_{i_{1}} x_{i_{2}} \cdots x_{i_{n}} .
$$

Here, the type B descent set, $\operatorname{Des}_{B}(\pi)$, keeps track of the ordinary descents as well as a descent in position 0 if $\pi_{1}<0$. Notice that if $\pi_{1}<0$, then $f\left(\pi_{1}\right)>0$, and $\Gamma_{B}(\pi)$ has no $x_{0}$ terms, as in

$$
\Gamma_{B}((-3,2,-1))=\sum_{0<i \leq j<k} x_{i} x_{j} x_{k}
$$


The possible presence of a descent in position zero is the crucial difference between type A and type B descent sets. Define a pseudo-composition of $n$ to be an ordered tuple $\alpha=\left(\alpha_{1}, \ldots, \alpha_{k}\right)$ with $\alpha_{1} \geq 0$, and $\alpha_{i}>0$ for $i>1$, such that $\alpha_{1}+\cdots+\alpha_{k}=n$. We write $\alpha \Vdash n$ to mean $\alpha$ is a pseudo-composition of $n$. Define the descent pseudo-composition $C_{B}(\pi)$ of a signed permutation $\pi$ be the lengths of its increasing runs as before, but now we have $\alpha_{1}=0$ if $\pi_{1}<0$. As with ordinary compositions, the partial order on pseudocompositions of $n$ is given by reverse refinement. We can move back and forth between descent pseudo-compositions and descent sets in exactly the same way as for type A. If $C_{B}(\pi)=\left(\alpha_{1}, \ldots, \alpha_{k}\right)$, then we have

$$
\operatorname{Des}_{B}(\pi)=\left\{\alpha_{1}, \alpha_{1}+\alpha_{2}, \ldots, \alpha_{1}+\alpha_{2}+\cdots+\alpha_{k-1}\right\} .
$$

We will use pseudo-compositions of $n$ to index the type B quasisymmetric functions. Define $\mathcal{B} \mathcal{Q}$ sym $_{n}$ as the vector space of functions spanned by the type $B$ monomial quasisymmetric functions:

$$
M_{B, \alpha}:=\sum_{0<i_{2}<\cdots<i_{k}} x_{0}^{\alpha_{1}} x_{i_{2}}^{\alpha_{2}} \cdots x_{i_{k}}^{\alpha_{k}}
$$

where $\alpha=\left(\alpha_{1}, \ldots, \alpha_{k}\right)$ is any pseudo-composition, or equivalently by the type $B$ fundamental quasisymmetric functions:

$$
F_{B, \alpha}:=\sum_{\alpha \preccurlyeq \beta} M_{B, \beta}
$$

The space of all type B quasisymmetric functions is defined as the direct sum $\mathcal{B} \mathcal{Q}$ sym $:=$ $\bigoplus_{n \geq 0} \mathcal{B} \mathcal{Q}$ sym $_{n}$. By design, we have

$$
\Gamma_{B}(\pi)=F_{B, C_{B}(\pi)}
$$

From Chow [4] we have the following theorem and corollary.

Theorem 2 As sets, we have the bijection

$$
\mathcal{A}_{B}(\pi ; S T) \leftrightarrow \coprod_{\sigma \tau=\pi} \mathcal{A}_{B}(\tau ; S) \oplus \mathcal{A}_{B}(\sigma ; T)
$$

where $S T$ is the cartesian product of the sets $S$ and $T$ with the lexicographic ordering.

We take $S=T=\mathbb{Z}$ and we have the following.

Corollary 2 We have

$$
F_{B, C_{B}(\pi)}(X Y)=\sum_{\sigma \tau=\pi} F_{B, C_{B}(\tau)}(X) F_{B, C_{B}(\sigma)}(Y)
$$


The coalgebra structure on $\mathcal{B Q}$ sym $_{n}$ works just the same as in the type A case. Corollary 2 gives us the coproduct

$$
F_{B, \gamma} \mapsto \sum_{\alpha, \beta \Vdash n} b_{\alpha, \beta}^{\gamma} F_{B, \beta} \otimes F_{B, \alpha},
$$

where for any $\pi$ such that $C_{B}(\pi)=\gamma, b_{\alpha, \beta}^{\gamma}$ is the number of pairs of signed permutations $(\sigma, \tau)$ such that $C_{B}(\sigma)=\alpha, C_{B}(\tau)=\beta$, and $\sigma \tau=\pi$. The dual algebra is isomorphic to $\operatorname{Sol}\left(B_{n}\right)$, where if $u_{\alpha}$ is the sum of all signed permutations with descent pseudo-composition $\alpha$, the multiplication given by

$$
u_{\beta} u_{\alpha}=\sum_{\gamma \Vdash n} b_{\alpha, \beta}^{\gamma} u_{\gamma}
$$

\section{Signed quasisymmetric functions and the Mantaci- Reutenauer algebra}

One thing to have noticed about the generating function for type B $P$-partitions is that we are losing a certain amount of information when we take absolute values on the subscripts. We can think of signed quasisymmetric functions as arising naturally by dropping this restriction.

For a type B poset $P$, define the signed generating function for type B $P$-partitions to be

$$
\bar{\Gamma}(P):=\sum_{f \in \mathcal{A}_{B}(P)} x_{f(1)} x_{f(2)} \cdots x_{f(n)}
$$

where we will write

$$
x_{i}= \begin{cases}u_{i} & \text { if } i<0 \\ v_{i} & \text { if } i \geq 0\end{cases}
$$

In the case where $P$ is a signed permutation, we have

$$
\bar{\Gamma}(\pi)=\sum_{\substack{0 \leq i_{1} \leq i_{2} \leq \ldots \leq i_{n} \\ s \in \operatorname{Des}_{B}(\pi) \Rightarrow i_{s}<i_{s+1} \\ \pi_{s}<0 \Rightarrow x_{s}=i_{s} \\ \pi_{s}>0 \Rightarrow x_{s_{s}}=v_{i_{s}}}} x_{i_{1}} x_{i_{2}} \cdots x_{i_{n}}
$$

so that now we are keeping track of the set of minus signs of our signed permutation along with the descents. For example,

$$
\bar{\Gamma}((-3,2,-1))=\sum_{0<i \leq j<k} u_{i} v_{j} u_{k}
$$

To keep track of both the set of signs and the set of descents, we introduce the signed compositions as used in [3]. A signed composition $\alpha$ of $n$, denoted $\alpha \| \vdash n$, is a tuple of nonzero integers $\left(\alpha_{1}, \ldots, \alpha_{k}\right)$ such that $\left|\alpha_{1}\right|+\cdots+\left|\alpha_{k}\right|=n$. For any signed 
permutation $\pi$ we will associate a signed composition $s C(\pi)$ by simply recording the length of increasing runs with constant sign, and then recording that sign. For example, if $\pi=$ $(-3,4,5,-6,-2,-7,1)$, then $s C(\pi)=(-1,2,-2,-1,1)$. The signed composition keeps track of both the set of signs and the set of descents of the permutation as we demonstrate with an example. If $s C(\pi)=(-3,2,1,-2,1)$, then we know that $\pi$ is a permutation in $\mathfrak{S}_{9}$ such that $\pi_{4}, \pi_{5}, \pi_{6}$, and $\pi_{9}$ are positive, whereas the rest are all negative. The descents of $\pi$ are in positions 5 and 6 . Note that for any ordinary composition of $n$ with $k$ parts, there are $2^{k}$ signed compositions, leading us to conclude that there are

$$
\sum_{k=1}^{n}\left(\begin{array}{l}
n-1 \\
k-1
\end{array}\right) 2^{k}=2 \cdot 3^{n-1}
$$

signed compositions of $n$. The partial order on signed compositions is given by reverse refinement with constant sign, i.e., the cover relations are still of the form:

$$
\left(\alpha_{1}, \ldots, \alpha_{i}+\alpha_{i+1}, \ldots, \alpha_{k}\right) \prec\left(\alpha_{1}, \ldots, \alpha_{i}, \alpha_{i+1}, \ldots, \alpha_{k}\right),
$$

but now $\alpha_{i}$ and $\alpha_{i+1}$ have to have the same sign. For example, if $n=2$, we have the following partial order:

$$
\begin{aligned}
& (2) \prec(1,1) \\
& (-1,1) \\
& (1,-1) \\
& (-2) \prec(-1,-1)
\end{aligned}
$$

We will use signed compositions to index the signed quasisymmetric functions (see [9]). For any signed composition $\alpha$, define the monomial signed quasisymmetric function

$$
\bar{M}_{\alpha}:=\sum_{\substack{i_{1}<i_{2}<\cdots<i_{k} \\ \alpha_{r}<0 \Rightarrow x_{i_{r}}=u_{i_{r}} \\ \alpha_{r}>0 \Rightarrow x_{i_{r}}=v_{i_{r}}}} x_{i_{1}}^{\left|\alpha_{1}\right|} x_{i_{2}}^{\left|\alpha_{2}\right|} \cdots x_{i_{k}}^{\left|\alpha_{k}\right|}
$$

and the fundamental signed quasisymmetric function

$$
\bar{F}_{\alpha}:=\sum_{\alpha \preccurlyeq \beta} \bar{M}_{\beta} .
$$

By construction, we have

$$
\bar{\Gamma}(\pi)=\bar{F}_{s C(\pi)} .
$$

Notice that if we set $u=v$, then our signed quasisymmetric functions become type B quasisymmetric functions.

Let $\mathcal{S} \mathcal{Q}$ sym $_{n}$ denote the span of the $\bar{M}_{\alpha}$ (or $\bar{F}_{\alpha}$ ), taken over all $\alpha \| \vdash n$. The space of all signed quasisymmetric functions, $\mathcal{S} \mathcal{Q}$ sym $:=\bigoplus_{n \geq 0} \mathcal{S} \mathcal{Q}$ sym $_{n}$, is a graded ring whose $n$-th graded component has rank $2 \cdot 3^{n-1}$. We will relate this to the Mantaci-Reutenauer algebra. 
Theorem 2 is a statement about splitting apart bipartite $P$-partitions, independent of how we choose to encode the information. So while Corollary 2 is one such way of encoding the information of Theorem 2, the following is another.

Corollary 3 We have

$$
\bar{F}_{s C(\pi)}(X Y)=\sum_{\sigma \tau=\pi} \bar{F}_{s C(\tau)}(X) \bar{F}_{s C(\sigma)}(Y)
$$

We define a coalgebra structure on $\mathcal{S} \mathcal{Q} \operatorname{sym}_{n}$ as we did in the earlier cases. Let $\pi \in \mathfrak{B}_{n}$ be any signed permutation with $s C(\pi)=\gamma$, and let $c_{\alpha, \beta}^{\gamma}$ be the number of pairs of permutations $(\sigma, \tau) \in \mathfrak{B}_{n} \times \mathfrak{B}_{n}$ with $s C(\sigma)=\alpha, s C(\tau)=\beta$, and $\sigma \tau=\pi$. Corollary 3 gives a coproduct $\mathcal{S} \mathcal{Q}$ sym $_{n} \rightarrow \mathcal{S} \mathcal{Q}$ sym $_{n} \otimes \mathcal{S Q}$ sym $_{n}$ :

$$
\bar{F}_{\gamma} \mapsto \sum_{\alpha, \beta \| n} c_{\alpha, \beta}^{\gamma} \bar{F}_{\beta} \otimes \bar{F}_{\alpha} .
$$

Multiplication in the dual algebra $\mathcal{S Q} s y m_{n}^{*}$ is given by

$$
\bar{F}_{\beta}^{*} * \bar{F}_{\alpha}^{*}=\sum_{\gamma \| \Vdash n} c_{\alpha, \beta}^{\gamma} \bar{F}_{\gamma}^{*} .
$$

The group algebra of the hyperoctahedral group, $\mathbb{Z} \mathfrak{B}_{n}$, has a dual coalgebra $\mathbb{Z} \mathfrak{B}_{n}^{*}$ with comultiplication given by the map

$$
\pi \mapsto \sum_{\sigma \tau=\pi} \tau \otimes \sigma
$$

By Corollary 3, the following is a surjective homomorphism of coalgebras $\psi^{*}: \mathbb{Z} \mathfrak{B}_{n}^{*} \rightarrow$ $\mathcal{S Q}$ sym $_{n}$ given by

$$
\psi^{*}(\pi)=\bar{F}_{s C(\pi)} .
$$

The dualization of this map is an injective homomorphism $\psi: \mathcal{S} \mathcal{Q} \operatorname{sym}_{n}^{*} \rightarrow \mathbb{Z} \mathfrak{B}_{n}$ with

$$
\psi\left(\bar{F}_{\alpha}^{*}\right)=\sum_{s C(\pi)=\alpha} \pi .
$$

The image of $\psi$ is then a subalgebra of $\mathbb{Z} \mathfrak{B}_{n}$ of dimension $2 \cdot 3^{n-1}$, with basis

$$
v_{\alpha}:=\sum_{s C(\pi)=\alpha} \pi .
$$

This subalgebra is called the Mantaci-Reutenauer algebra [6], with multiplication given explicitly by

$$
v_{\beta} v_{\alpha}=\sum_{\gamma \| r n} c_{\alpha, \beta}^{\gamma} v_{\gamma}
$$

The duality between $\mathcal{S} \mathcal{Q} \operatorname{sym}_{n}$ and the Mantaci-Reutenauer algebra was shown in [1], and the bases $\left\{\bar{F}_{\alpha}\right\}$ and $\left\{v_{\alpha}\right\}$ are shown to be dual in [2], but the the $P$-partition 
approach to the problem is new. As the Mantaci-Reutenauer algebra is defined for any wreath product $C_{m}<\mathfrak{S}_{n}$, i.e., any " $m$-colored" permutation group, it would be nice to develop a theory of colored $P$-partitions to tell the dual story in general.

In closing, we remark that this same method was put to use in [8], where Stembridge's enriched $P$-partitions [13] were generalized and put to use to study peak algebras. Variations on the theme can also be found in [7].

\section{References}

[1] P. Baumann and C. Hohlweg, A Solomon descent theory for the wreath products $G$ ᄂ $S_{n}$, arXiv: math.CO/0503011.

[2] N. Bergeron and C. Hohlweg, Coloured peak algebras and hopf algebras, arXiv: math.AC/0505612.

[3] C. Bonnafé and C. Hohlweg, Generalized descent algebra and construction of irreducible characters of hyperoctahedral groups, arXiv: math.CO/0409199 .

[4] C.-O. Chow, Noncommutative symmetric functions of type B, Ph.D. thesis, MIT (2001).

[5] I. Gessel, Multipartite P-partitions and inner products of skew Schur functions, Contemporary Mathematics 34 (1984), 289-317.

[6] R. Mantaci and C. Reutenauer, A generalization of Solomon's algebra for hyperoctahedral groups and other wreath products, Communications in Algebra 23 (1995), $27-56$.

[7] T.K. Petersen, Cyclic descents and P-partitions, to appear in Journal of Algebraic Combinatorics.

[8] T.K. Petersen, Enriched P-partitions and peak algebras, arXiv: math.CO/0508041.

[9] S. Poirier, Cycle type and descent set in wreath products, Discrete Mathematics 180 (1998), 315-343.

[10] L. Solomon, A Mackey formula in the group ring of a finite Coxeter group, Journal of Algebra 41 (1976), 255-264.

[11] R. Stanley, Enumerative Combinatorics, Volume I, Wadsworth \& Brooks/Cole, 1986.

[12] R. Stanley, Enumerative Combinatorics, Volume II, Cambridge University Press, 2001.

[13] J. Stembridge, Enriched P-partitions, Transactions of the American Mathematical Society 349 (1997), 763-788. 